\title{
EQUIPE EDITORIAL
}

\section{Editora-chefe}

Heloisa Monteiro Rosário, Universidade Federal do Rio Grande do Sul (UFRGS), Brasil

\section{Comissão Editorial}

Heloisa Monteiro Rosário, Universidade Federal do Rio Grande do Sul (UFRGS), Brasil Valdir do Nascimento Flores, Universidade Federal do Rio Grande do Sul (UFRGS), Brasil Claudia Luiza Caimi, Universidade Federal do Rio Grande do Sul (UFRGS), Brasil Sara Luiza Hoff, Universidade Federal do Rio Grande do Sul (UFRGS), Brasil

\section{Conselho Editorial}

Ângela Fernandes, Faculdade de Letras da Universidade de Lisboa (FLUL), Portugal

Carlos Alexandre Baumgarten, Pontifícia Universidade Católica do Rio Grande do Sul (PUCRS), Brasil

Eduardo Guimarães, Universidade Estadual de Campinas (Unicamp), Brasil

Eni P. Orlandi, Universidade Estadual de Campinas (Unicamp)/Universidade do Vale do Sapucaí (Univas), Brasil

Ernesto José Rodrigues, Universidade de Lisboa (ULisboa), Portugal

Flávio Ribeiro de Oliveira, Universidade Estadual de Campinas (Unicamp), Brasil

Freda Indursky, Universidade Federal do Rio Grande do Sul (UFRGS), Brasil

Jacyntho Lins Brandão, Universidade Federal de Minas Gerais (UFMG), Brasil

Jaime Ginzburg, Universidade de São Paulo (USP), Brasil

Leda Bisol, Pontifícia Universidade Católica do Rio Grande do Sul (PUCRS), Brasil

Leo Wetzels, Vrije Universiteit Amsterdam (VU), Holanda

María Alejandra Vitale, Universidad de Buenos Aires (UBA), Argentina

Marília dos Santos Lima, Universidade do Vale do Rio dos Sinos (Unisinos), Brasil

Vanessa Castagna, Università Ca' Foscari Venezia (UNIVE), Itália

Ricardo Barberena, Pontifícia Universidade Católica do Rio Grande do Sul (PUCRS), Brasil

Rita Terezinha Schmidt, Universidade Federal do Rio Grande do Sul (UFRGS), Brasil 
Robert Ponge, Instituto de Letras da Universidade Federal do Rio Grande do Sul (UFRGS), Brasil

Sylvie Vandaele, Université de Montreal (UdeM), Canadá

Teresa Cristina Cerdeira, Universidade Federal do Rio de Janeiro (UFRJ), Brasil

Thomas Johnen, Westsächsische Hochschule Zwickau Fakultät Angewandte Sprachen und Interkulturelle Komunikation (WHZ), Alemanha

Vilson José Leffa, Universidade Católica de Pelotas (UCPel), Brasil

Zilá Bernd, Universidade Federal do Rio Grande do Sul (UFRGS)/Centro Universitário Ritter dos Reis (UniRitter)

\section{Revisores}

Adriele Albuquerque de Souza, Universidade Federal do Rio Grande do Sul (UFRGS), Brasil Eduardo Belmonte de Souza, Universidade Federal do Rio Grande do Sul (UFRGS), Brasil Filipe Cieslak, Universidade Federal do Rio Grande do Sul (UFRGS), Brasil Hannah Kahn, Universidade Federal do Rio Grande do Sul (UFRGS), Brasil Luana Tiburi Dani Gauer, Universidade Federal do Rio Grande do Sul (UFRGS)/Instituto Federal do Rio Grande do Sul (IFRS), Brasil

Lucas Neves Costa, Universidade Federal do Rio Grande do Sul (UFRGS), Brasil Maria Petrucci Sperb, Universidade Federal do Rio Grande do Sul (UFRGS), Brasil Nathália da Silveira Martins, Universidade Federal do Rio Grande do Sul (UFRGS), Brasil Raphaela Monteiro Chittolina, Universidade Federal do Rio Grande do Sul (UFRGS), Brasil Sara Luiza Hoff, Universidade Federal do Rio Grande do Sul (UFRGS), Brasil

Vicente de Souza Cardoso Júnior, Universidade Federal do Rio Grande do Sul (UFRGS), Brasil

Vitor Fernandes, Universidade Federal do Rio Grande do Sul (UFRGS), Brasil

\section{Bolsista}

Lilianne Dias Souza, Universidade Federal do Rio Grande do Sul (UFRGS), Brasil

\section{Layout}

Lilianne Dias Souza, Universidade Federal do Rio Grande do Sul (UFRGS), Brasil Sara Luiza Hoff, Universidade Federal do Rio Grande do Sul (UFRGS), Brasil 


\section{Capa}

Leandro Bierhals Bezerra

DOI: https://dx.doi.org/10.22456/2238-8915.117857

Organon, Porto Alegre, v. 36, n. 71, p. 2-4, jan./jun. 2021.

DOI: $10.22456 / 2238-8915.117857$ 